\title{
Commentary: Distinct Domains in The Matricellular Protein, Lonely Heart, Are Crucial for Cardiac Extracellular Matrix Formation and Heart Function in Drosophila
}

\author{
Yanina Post, Achim Paululat*
}

University of Osnabrueck, Faculty of Biology \& Chemistry, Zoology and Developmental Biology, Barbarastraße 11, 49076 Osnabrueck, Germany

Article Info

\section{Article Notes}

Received: July 10, 2018

Accepted: September 18, 2018

\section{${ }^{*}$ Correspondence:}

Prof. Achim Paululat, University of Osnabrueck, Faculty of Biology \& Chemistry, Zoology and Developmental Biology, Barbarastraße 11, 49076 Osnabrueck, Germany; Telephone No: ++ (0)541 969-2861; Fax No: ++ (0)541 969-2587;

E-mail: Paululat@Biologie.Uni-Osnabrueck.DE.

C 2018 Paululat A. This article is distributed under the terms of the Creative Commons Attribution 4.0 International License.

\section{Key words}

ADAMTS proteins

Drosophila heart mode

Extracellular matrix

Collagen

Lonely heart

Pericardin

\section{Abstract}

It is well known that cardiovascular diseases are becoming the number one cause of mortality in developed countries ${ }^{1}$. Minimal differences in a wide range of biological pathways can lead to organ malfunction or failure. In addition to the heart itself, its surroundings are also crucial for proper organ integrity and functionality. Changes in extracellular matrix (ECM) composition, such as a higher amount of incorporated collagens, can result in cardiac hypertrophy ${ }^{2}$ or hypertension-related diastolic dysfunction ${ }^{3}$ by stiffening the surrounding matrix and constricting the embedded organ. Similar observations have been made while studying the heart of the fruit fly, Drosophila melanogaster. In this model organism, altered amounts of ECM components, including Laminin or Collagen IV (Viking), cause impaired cardiac function ${ }^{4}$. This demonstrates the notable relevance of an organ-specific ECM composition that provides the correct balance of stiffness and elasticity, both in vertebrates and invertebrates. In our recent study we were able to unravel the function of several protein domains belonging to the ADAMTS-like protein Lonely heart ${ }^{5}$. Furthermore, we analysed its interaction with the Collagen IV-like protein Pericardin and described new interactions within the extracellular matrix that led to a more detailed model of the whole network. In addition, we investigated the impact of altered Pericardin levels on physiological aspects, including locomotion and heart beating parameters, and strengthened the hypothesis that ECM composition is crucial for proper organ functionality. Here we summarize our approaches and comment on additional observations, possible follow-up analyses and restrictions as well as advantages. Moreover, we discuss possible additional roles of ADAMTS-like proteins within an extracellular matrix.

In Drosophila, the cardiac ECM anchors the heart within the body cavity and forms a dense network of adapter, linker and structural proteins, thereby rendering it able to withstand the strong mechanical forces that are applied during lifelong heart beating activity.

While most components of the cardiac ECM are also incorporated into other matrices and considered the basic core constituents, two proteins present in the Drosophila cardiac matrix are unique. One of them is Lonely heart (Loh), an ADAMTS-like adaptor protein ${ }^{6}$. It harbours several domains characteristic to the ADAMTS family while lacking the proteolytically active sequence (see Figure 1A). The other unique component is a Collagen IV-like protein named Pericardin (Prc) ${ }^{7}$. It relies heavily on Loh's activity to be recruited towards the cardiac matrix, where it starts meshwork formation.

We have previously shown that Loh is synthesised by heart cells and afterwards secreted and displayed (self-anchored) within the ECM. Once incorporated into the matrix, Loh is fully capable of recruiting Prc from the hemolymph towards the heart, where 
A

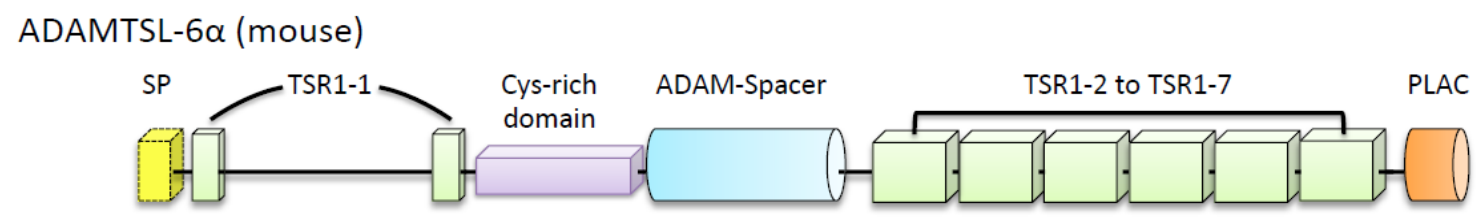

Lonely heart (Drosophila)

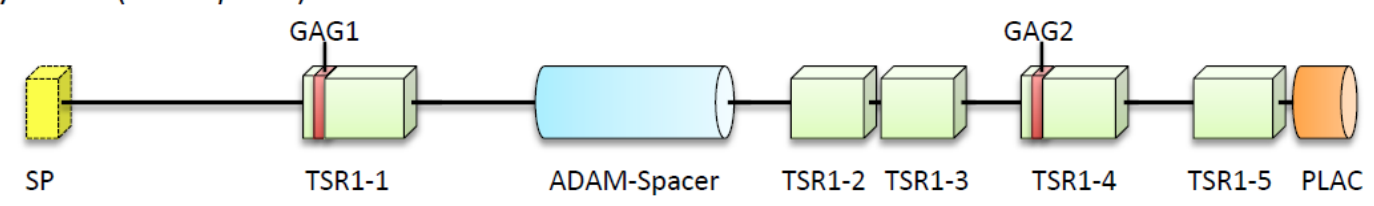

B

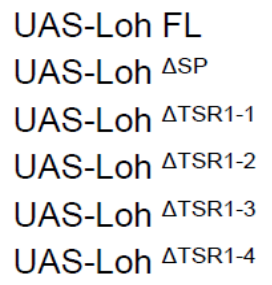

UAS-Loh $\triangle \mathrm{TSR} 1-5$

UAS-Loh $\triangle$ PLAC

UAS-Loh GAG1*

UAS-Loh GAG2*

UAS-Loh GAG1 $^{\star}+2^{\star}$

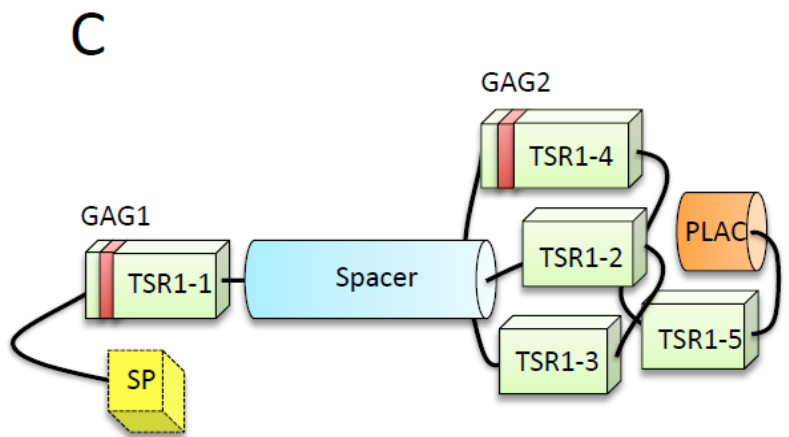

Figure 1: Schematic representation of ADAMTSL-6a (modified after [9]) in comparison to Lonely heart and the created deletion constructs (modified after [5]). (A) Schematic view of the predicted domain arrangement of ADAMTSL-6 $\alpha$ from mouse embryos and Lonely heart (Loh) from Drosophila melanogaster. The red bars within TSR1-1 and TSR1-4 of Loh indicate the presence of a putative glycosaminoglycan-binding site (GAG1 and GAG2). (B) Deletion constructs that have been tested in our recent study. Triangles indicate a deletion of the following domain, while asterisks indicate a modification of the respective motif. (C) Most probable in vivo arrangement of Lonely heart's protein domains based on protein modelling tools (Modified after [5]). SP = Signal peptide, TSR1= Thrombospondin type 1 repeat, GAG = putative glycosaminoglycan binding site, PLAC = Protease and Lacunin domain.

meshwork formation is initiated ${ }^{6}$. Prc itself is secreted by other cells, mainly adipocytes, and thereby released into the circulating hemolymph stream to be attracted towards target tissues by special anchor proteins, with Loh being the first protein identified with this functionality. Importantly, Loh mutants suffer from a de-stabilized cardiac matrix architecture, where continuous heart beating leads to a disruption of the ECM upon ageing of the individual, resulting in a total loss of heart integrity and circulatory force.

In our recent study, we aimed to answer the questions: 1) which protein domains mediate Loh self-anchoring within the ECM; and 2) which protein domains are essential for Prc recruitment? As the Drosophila genome features only one ADAMTS-like protein (Loh)[6], while in mammalians there are seven members known ${ }^{8}$, Drosophila is a perfect model organism for such an in vivo analysis. Furthermore, a domain comparison between Loh and vertebrate ADAMTS-like proteins revealed that there is a high similarity between Loh and the ADAMTSL- $6 \alpha$ (see
Fig. 1A), first described in mouse embryos. ADAMTSL- $6 \alpha$ is an extracellular protein involved in fibrillin-1 binding and fibrillin-1 matrix assembly ${ }^{9}$, two characteristic functions resembling the tasks Loh mediates. A lack of ADAMTSL$6 \alpha$ leads to severe diseases like the Marfan syndrome, a systemic disorder, which is mainly characterised by a malformation of the fibrillin-1 network ${ }^{10}$.

A range of Loh mutants have been generated that lack single domains or motifs of the protein (see Fig. 1B). By expressing them in insect cell culture or crossing respective fly strains with particular driver lines, we investigated the secretion of the deletion constructs and their ability to anchor within the matrix. As a second step, we assessed the capability of every construct to recruit Prc from the hemolymph towards an ECM.

We identified the first thrombospondin repeat (TSR11) and the included putative glycosaminoglycan-binding site (GAG1) as being vital for anchorage of Loh within the matrix. While a deletion of the signal peptide led to an intracellular accumulation of the protein, the deletion 
of the first TSR1 or a modification of the included GAGbinding site resulted in a secreted protein that is no longer capable of binding to the matrix. A similar observation has been made for the construct where both GAG-binding sites were altered, though in this case, the protein is trapped within the secretory pathway. A possible explanation lies in the deletion of putative glycosylation sites, including a C-mannosylation and $\mathrm{O}$-fucosylation site, which were predicted within the GAG motifs. It has been shown that a modified glycosylation pattern increases the risk of protein instability or results in an unsuccessful secretion ${ }^{11}$. As all additionally tested Loh constructs were successfully secreted and anchored properly within the matrix, this problem seems only to account for a deletion of both respective glycosylation sites.

Furthermore, we discovered that two different TSR1s mediate the recruitment of Prc towards an ECM and the induction of network formation. The Prc network density was significantly reduced in animals expressing the TSR12 deletion construct or the TSR1-4 construct. In addition also a modification of the second GAG binding site (GAG2) lead to the same result. This indicates that different domains of Loh mediate anchoring (TSR1-1 and GAG1) and recruitment processes (TSR1-2, TSR1-4 and GAG2), while the functions of other domains remain unclear.

In addition, we tested whether different matrices harbour the intrinsic capacity to incorporate these proteins and develop respective networks. We induced the expression of full-length Loh on adipocytes, parts of the wing disc, the central nervous system (CNS) and salivary glands. All tissues successfully expressed, secreted and incorporated Loh into their matrices, but, interestingly, the recruitment capacity of the different cell types varied. While we noted dense networks of Prc on adipocytes, the wing disc and the CNS, Loh at the salivary glands failed to recruit Prc. This outcome may point towards the necessity of another factor that facilitates proper Prc incorporation or regulates Loh's functionality. As a follow-up analysis a comparison of the different matrisomes would be a powerful tool to gain new insights into the individual ECM compositions. Thereby also another crucial factor for Prc recruitment may be discovered that would explain the observed phenotype. One possibility is an interaction of Prc with Integrins, as Prc harbours an RGD motif [7]. This triplet of amino acids has been shown to be involved in the binding of Integrins ${ }^{12}$, while its functionality in Prc still has to be analysed.

Additional observations indicate that Prc is able to bind (at a much lower efficiency) to a matrix, even in the absence of Loh. We observed small networks or attached fibres of Prc on body wall muscles or adipocytes that do not display Loh. This suggests a somehow intrinsic capacity of Prc to stick to different matrices by itself. This connection is most likely weaker in the absence of Loh and may not withstand strong mechanical forces, but it still occurs. It has been shown that Prc is highly glycosylated and it is possible that the attached proteoglycans mediate this selfattachment ${ }^{13}$. Western Blot analysis revealed that Prc coimmunoprecipitates with a variety of ECM components, excluding ßPS-Integrin, which was most likely due to a missfolding of the used Integrin:GFP construct. Nevertheless, the interactions with Laminin A (LanA), Laminin B1 (LanB1) and Viking (Vkg) provide new insights into the interconnection of the extracellular network (see Figure 2) and may hold an additional explanation for the self-attachment of Prc to a matrix.

However, the presence of Loh increased the assembly of Prc substantially, illustrating the existence of a new regulatory mechanism to generate tissue-specific matrix assembly and matrix composition, which is required for building up specific biomechanical properties of the tissues, e.g., display the correct balance of stiffness and elasticity needed for flexible organs. A follow-up analysis uncovered that Prc was only present where Loh was expressed and did not spread to adjacent parts. This supports our theory that the interaction between Loh and Prc is highly specific.

An ultrastructural analysis revealed that third instar larvae overexpressing Loh exhibited large accumulations of ECM material all over the heart. Furthermore, we conducted a semi-intact optical analysis of the heartbeat (SOHA) ${ }^{17}$. Thereby, we were able to detect a significantly decreased heart rate in the Loh overexpression line and a significantly elevated heart rate for the Loh null mutant. In addition, the arrhythmia indices for both lines were significantly increased compared to the control group. These data suggest that the right balance between distinct ECM components is critical for proper organ functionality. Increasing the amount of Prc results in a constriction of the heart and reduces the heart rate, while a decrease of Prc shows the opposite effect ${ }^{5}$.

\section{Discussion}

In our recent study, we unravelled the function of several Loh protein domains and detected new interconnections within the cardiac ECM of Drosophila. However, Loh harbours evolutionary conserved protein domains with still unknown functions, e.g., the so-called PLAC domain, a short C-terminal motif present in proteins like Perlecan and others, including Loh. Until now, the functional role of the PLAC domain remains elusive.

Furthermore, we provided evidence that the spacer region of the ADAMTS-like protein is presumably essential for protein stability. A deletion construct was degraded right after translation and reviewing the predicted threedimensional (3D) model, it became obvious that the bridge between the first TSR1 and the last four TSR1s was 


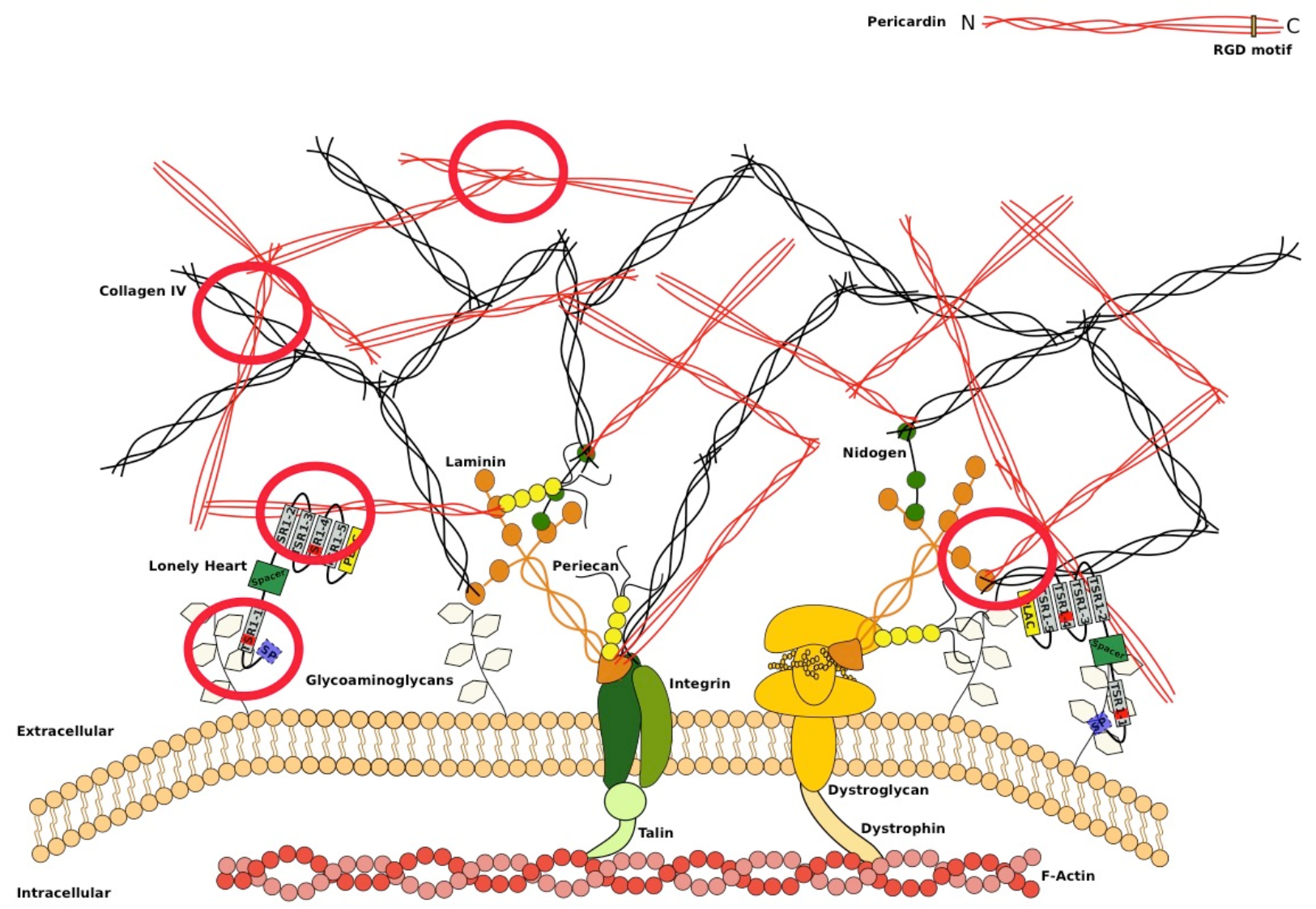

Figure 2: Schematic representation of an extracellular matrix (ECM) with incorporated Pericardin (Prc) Transmembrane receptors, like Integrins and Dystroglycans, link the ECM to the actin cytoskeleton ${ }^{14}$. Additionally, connections between the large structural ECM constituents, including Collagen IV, Prc and Integrin/Dystroglycan, are formed by the presence of Laminins ${ }^{15}$. Furthermore several linker proteins like Perlecan (in Drosophila named Trol) connect for example Collagens to Laminins ${ }^{16}$. Loh is depicted as a protein-domain scheme, based on protein modelling tools, with a signal peptide indicated in light blue, the ADAMTS-spacer region in green, the PLAC domain in yellow and the remaining thrombospondin type-1 repeats in grey, of which two harbour a GAG-binding site (red bars). The position of Loh is based on our results and indicates that it binds to glycosaminoglycans of membrane-associated proteins via its first GAG-binding site and to Prc (directly or indirectly) via its second and fourth thrombospondin type-1 repeat. Red circles signify the newly confirmed interactions based on data published in this article (modified from [5]).

potentially critical for the protein's stability and folding.

It has been put forth for ADAMTS proteins that they are involved in the cleavage of collagens ${ }^{18,19}$, so it is indeed possible that ADAMTSL proteins also have a collagenrecognition site within the spacer region, but as they lack the proteolytically active sequence, they may only bind the collagens without modifying them.

Although we were able to demonstrate that certain domains of Loh are crucial for Prc recruitment, a direct interaction - if it exists - still lacks proof. It is also possible that an additional protein acts as a mediator between Loh and Prc, or that Loh regulates the function of a Prc binding protein. It has been shown for ADAMTSL proteins that they have an enhancing or supressing function on other ADAMTS proteins ${ }^{20}$. Drosophila Papilin can for example non-competitively inhibit bovine ADAMTS $2^{21}$. One might speculate that in case of Loh the second TSR1 might not be directly involved in Prc recruitment, but rather interacts with another protein or protease, which then recruits, anchors or modifies Prc.

In general we provided a model system that allows an easy analysis of protein interactions in vivo. Thereby it is assured that our proteins of interest display all secondary modifications that are needed for proper functionality. This is a special advantage in case of ECM proteins, as their glycosylation level may have an impact on selfassembly and incorporation into a matrix that cannot be studied in cell culture systems. Furthermore, our analysis is conducted in a whole-tissue-context, which gives us the additional assurance that all proteins behave in their native manner.

Nevertheless, all studies including living organisms face similar problems. There are always individual differences between animals and their impact has to be reduced to a 
minimum. In our case the performed co-immunostainings with F-actin as an intensity marker were used as an indicator of differences in fixation and staining procedure. In addition also different groups of muscle tissue may behave differently and although our observations suggest a general capability of muscles to incorporate Loh and recruit Prc, our results do not have to account for every muscle strain.

\section{Conclusion}

In our recent work, we showed that specific domains of the ADAMTS-L protein, Lonely heart (Loh), manage its anchorage within the cardiac extracellular matrix (ECM) and the recruitment of the Collagen IV-like protein, Pericardin (Prc) in Drosophila melanogaster.

Increasing or decreasing the amount of Loh and Prc in vivo resulted in altered heart and body wall muscle performance, indicating that the proper balance of strength versus elasticity of ECMs is of crucial relevance and, regarding the cardiac ECM, relies significantly on the correct amount of these two components. We provided evidence that their interaction is highly specific, but seems to involve another as-of-yet unknown factor. The results of our immunoprecipitation assay indicate that Prc interacts with itself and additional ECM components, including LanA, LanB1 and Vkg. The identification of these new interaction partners of Prc and the analysis of the interactions between Prc and Loh lead to a more detailed model of the cardiac matrix of Drosophila.

\section{Financial Support}

This work was supported by grants from the Deutsche Forschungsgemeinschaft to A.P. (PA 517/12-1, SFB 944), the DAAD (IPID program) and from the State of LowerSaxony (ZN2832).

\section{References}

1. Lopez AD, Mathers CD, Ezzati M, et al. Global burden of disease and risk factors. Oxford University Press. 2006.

2. Bishop JE, Laurent GJ. Collagen turnover and its regulation in the normal and hypertrophying heart. Eur Heart J. 1995; 16 Suppl C: 3844.

3. Brower GL, Gardner JD, Forman MF, et al. The relationship between myocardial extracellular matrix remodeling and ventricular function. Eur J Cardiothorac Surg. 2006; 30(4): 604-610.

4. Sessions AO, Kaushik G, Parker S, et al. Extracellular matrix downregulation in the Drosophila heart preserves contractile function and improves lifespan. Matrix Biol. 2017; 62: 15-27.

5. Rotstein B, Post Y, Reinhardt M, et al. Distinct domains in the matricellular protein Lonely heart are crucial for cardiac extracellular matrix formation and heart function in Drosophila. J Biol Chem. 2018; 293(20): 7864-7879.

6. Drechsler M, Schmidt AC, Meyer H, et al. The conserved ADAMTS-like protein Lonely heart mediates matrix formation and cardiac tissue integrity. PLoS Genetics. 2013; 9(7): e1003616.

7. Chartier A, Zaffran S, Astier M, et al. Pericardin, a Drosophila type IV collagen-like protein is involved in the morphogenesis and maintenance of the heart epithelium during dorsal ectoderm closure. Development. 2002; 129(13): 3241-3253.

8. Dubail J, Apte SS. Insights on ADAMTS proteases and ADAMTS-like proteins from mammalian genetics. Matrix Biol. 2015; 44-46: 24-37.

9. Tsutsui K, Manabe R, Yamada $T$, et al. ADAMTSL-6 is a novel extracellular matrix protein that binds to fibrillin-1 and promotes fibrillin-1 fibril formation. J Biol Chem. 2010; 285(7): 4870-4882.

10. Saito M, Kurokawa M, Oda M, et al. ADAMTSL6 beta\} rescues fibrillin-1 microfibril disorder in Marfan syndrome mouse model through the promotion of fibrillin-1 assembly. J Biol Chem. 2011; 286(44): 38602 38613.

11. Wang LW, Dlugosz M, Somerville RP, et al. O-fucosylation of thrombospondin type 1 repeats in ADAMTS-like-1/punctin-1 regulates secretion: implications for the ADAMTS superfamily. J Biol Chem. 2007; 282(23): 17024-17031.

12. Ruoslahti E. RGD and other recognition sequences for integrins. Annu Rev Cell Dev Biol. 1996; 12: 697-715.

13. Wilmes AC, Klinke N, Rotstein B, et al. Biosynthesis and assembly of the Collagen IV-like protein Pericardin in Drosophila melanogaster. Biol Open. 2018; 7(4): pii: bio030361.

14. Hynes RO. Integrins: versatility, modulation, and signaling in cell adhesion. Cell. 1992; 69(1): 11-25.

15. Hynes RO, Yamada KM. Extracellular Matrix Biology. Cold Spring Harbor perspectives in biology. Cold Spring Harbor, N.Y.: Cold Spring Harbor Laboratory Press. 2012.

16. Friedrich MV, Schneider M, Timpl R, et al. Perlecan domain V of Drosophila melanogaster. Sequence, recombinant analysis and tissue expression. Eur J Biochem. 2000; 267(11): 3149-3159.

17. Fink M, Callol-Massot C, Chu A, et al. A new method for detection and quantification of heartbeat parameters in Drosophila, zebrafish, and embryonic mouse hearts. Biotechniques. 2009; 46(2): 101-113.

18. Colige A, Beschin A, Samyn B, et al. Characterization and partial amino acid sequencing of a 107-kDa procollagen I N-proteinase purified by affinity chromatography on immobilized type XIV collagen. J Biol Chem. 1995; 270(28): 16724-16730.

19. Fernandes RJ, Hirohata S, Engle JM, et al. Procollagen II amino propeptide processing by ADAMTS-3. Insights on dermatosparaxis. J Biol Chem. 2001; 276(34): 31502-31509.

20. Apte SS. A disintegrin-like and metalloprotease (reprolysin-type) with thrombospondin type 1 motif (ADAMTS) superfamily: functions and mechanisms. J Biol Chem. 2009; 284(46): 31493-31497.

21. Kramerova IA, Kawaguchi N, Fessler LI, et al. Papilin in development; a pericellular protein with a homology to the ADAMTS metalloproteinases. Development. 2000; 127(24): 5475-5485. 